\title{
Fast Method of Sparse Acquisition and Reconstruction of View and Illumination Dependent Datasets
}

\author{
Jiří Filip, Radomír Vávra \\ Institute of Information Theory and Automation of the AS CR, Prague, Czech Republic
}

\begin{abstract}
Although computer graphics uses measured view and illumination dependent data to achieve realistic digital reproduction of realworld material properties, the extent of their utilization is currently limited by a complicated acquisition process. Due to the high dimensionality of such data, the acquisition process is demanding on time and resources. Proposed is a method of approximate reconstruction of the data from a very sparse dataset, obtained quickly using inexpensive hardware. This method does not impose any restrictions on input datasets and can handle anisotropic, non-reciprocal view and illumination direction-dependent data. The method's performance was tested on a number of isotropic and anisotropic apparent BRDFs, and the results were encouraging. The method performs better than the uniform sampling of a comparable sample count and has three main benefits: the sparse data acquisition can be done quickly using inexpensive hardware, the measured material does not need to be extracted or removed from its environment, and the entire process of data reconstruction from the sparse samples is quick and reliable. Finally, the ease of sparse dataset acquisition was verified in measurement experiments with three materials, using a simple setup of a consumer camera and a single LED light. The proposed method has also shown promising performance when applied to sparse measurement and reconstruction of BTFs, mainly for samples with a lower surface height variation. Our approach demonstrates solid performance across a wide range of view and illumination dependent datasets, therefore creating a new opportunity for development of time and cost-effective portable acquisition setups.
\end{abstract}

Keywords: apparent BRDF, measurement, reconstruction, sparse sampling, portable setup, BTF

\section{Introduction}

2 View and illumination dependent data can be beneficial in з many computer graphic applications, due to their ability to dig4 itally represent the actual appearance of respective material. 5 However, their measurement is costly and time-consuming, be6 cause standard acquisition procedures of such data often require 7 lengthy measurements, or either a specific shape of the mea8 sured sample or a dedicated measurement setup. Bidirectional 9 reflectance distribution function (BRDF) [25], spatially varying ${ }_{10} \mathrm{BRDF}$ (SV-BRDF) and bidirectional texture function (BTF) [3] 11 are examples of such data. While a four-dimensional BRDF de12 scribes distribution of energy reflected to the viewing direction 13 when illuminated from a specific direction, a six-dimensional ${ }_{14}$ SVBRDF additionally captures the spatial dependency of re15 flectance across a material surface. While BRDF and SVBRDF ${ }_{16}$ impose restrictions on reciprocity, opacity and a range of sam17 ple height variations, the six-dimensional BTF generally does 18 not fulfill these restrictions. This is due to local effects in a 19 rough material structure such as occlusions, masking, subsur20 face scattering, and inter-reflections.

Therefore, individual BTF pixels are not regarded as BRDF 22 but rather apparent BRDF (ABRDF). If we process individ-

Email addresses: filipj@utia.cas.cz (Jiří Filip), vavra@utia.cas.cz (Radomír Vávra)

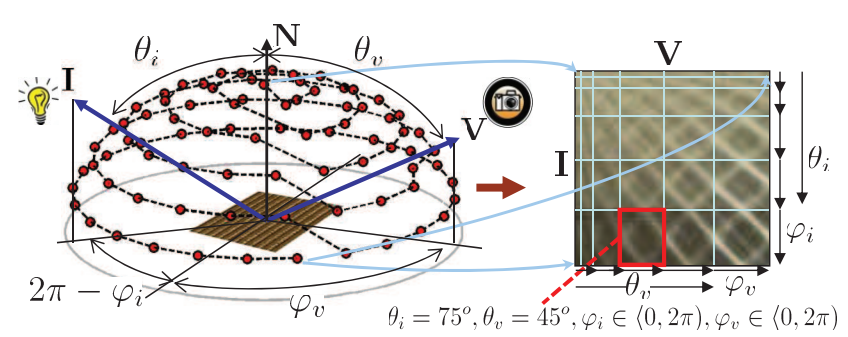

Figure 1: Parameterization (left) of view and illumination-dependent data of a single/average pixel (right).

${ }_{23}$ ual color/spectral channels separately, the ABRDF can be rep24 resented by a four-dimensional function $\operatorname{ABRDF}\left(\theta_{i}, \varphi_{i}, \theta_{v}, \varphi_{v}\right)$. ${ }_{25} \mathrm{ABRDF}$ is the most general data representation of a reflectance 26 of opaque materials dependent on local illumination $\mathbf{I}\left(\theta_{i}, \varphi_{i}\right)$ ${ }_{27}$ and view $\mathbf{V}\left(\theta_{v}, \varphi_{v}\right)$ directions; therefore, we focus on its proper 28 acquisition and reconstruction in this paper. Its typical param29 eterization by elevation $\theta$ and azimuthal $\varphi$ angles is shown in ${ }_{30}$ Fig. 1-left. A projection of the 4D ABRDF, representing de${ }_{31}$ pendence of view and illumination directions of a single pixel ${ }_{32}(\mathrm{BTF})$ or its average value (BRDF) by means of a 2D image, is ${ }_{33}$ shown in Fig. 1-right. Note that individual rectangles (an exam${ }_{34}$ ple is shown in red) represent $2 \mathrm{D}$ subspaces of $4 \mathrm{D}$ ABRDF at ${ }_{35}$ constant elevations $\left(\theta_{i} / \theta_{v}\right)$. These subspaces are toroidal. That ${ }_{36}$ is data of the highest $\varphi \approx 2 \pi$ are followed by data of the lowest ${ }_{37} \varphi \approx 0$. 
Main contributions of this paper:

- a reconstruction method of the entire anisotropic ABRDF space from less than two hundred sparsely measured samples

- a practically verified novel method for intuitive and fast ABRDF acquisition and reconstruction using a consumer camera and LED light in under 10 minutes.

\section{Main features of the proposed method:}

- a correct reconstruction of non-reciprocal, energy nonconserving ABRDF data

- an arbitrarily dense sampling of specular highlights, without increasing measurement time

- no need for lengthy measurement using a dedicated and expensive measurement setup

- not necessary to process or extract the measured sample from its environment

- contrary to analytical BRDF models, this method requires neither a lengthy fitting procedure nor guessing at initialization values.

40 This paper is structured as follows: Section 2 sets work in ${ }_{41}$ the context of related research. Section 3 explains the princi${ }_{42}$ ple of the proposed method. Section 4 shows results of per${ }_{43}$ formed experiments. The method's limitations are discussed in ${ }_{44}$ Section 5, and pilot project results of the real data acquisition ${ }_{45}$ scenario are shown in Section 6. Section 7 shows experimen${ }_{46}$ tal reconstruction of BTF samples, and Section 8 concludes the ${ }_{47}$ paper.

\section{${ }_{48}$ 2. Prior Work}

49 The proposed work relates to methods of BRDF or SVBRDF ${ }_{50}$ acquisition and interpolation from sparse samples.

Such data were initially captured by setups based on go${ }_{52}$ nioreflectometers realizing a required four mechanical degrees ${ }_{53}$ of freedom (DOF) of camera/light/sample movement [12], [29]. ${ }_{54}$ Because measurement times were too long, setups were used ${ }_{55}$ which reduced the required number of DOF using parabolic ${ }_{56}$ mirrors [4], or kaleidoscope [10]. They allowed the capture 57 of many viewing directions simultaneously; however a limited 58 range of surface height or elevation angles resulted. Measure59 ment time can also be reduced by using multiple lights and sen${ }_{60}$ sors simultaneously [20]; yet, high financial cost is associated ${ }_{61}$ with such a setup. Another group of fast acquisition methods 62 reduces the number of DOF by using a known shape of the 63 sample [19], [32], [17], [23], [13]. However, these approaches ${ }_{64}$ are often limited to isotropic BRDFs, or focus on represent65 ing sparsely sampled data using a parametric BRDF model. ${ }_{66}$ There is an existing statistical acquisition approach [22] allow67 ing quick and economical measurement of ABRDF; however, it 68 requires several samples of material with regular structure, po69 sitioned in different orientations with respect to the camera. Un70 fortunately, methods [22],[23] require a specific sample shape 71 or placement coupled with its extraction from the original envi72 ronment. Isotropic SVBRDF can also be estimated from pho73 tometric stereo using a parametric reflectance model [9] or bi74 variate BRDF [1]. Finally, it is possible to use portable setups
75 measuring SVBRDF by matching sparsely locally measured 76 isotropic BRDFs (using condenser lens optics) with sparsely 77 measured global reflectance fields [6]. Another approach records ${ }_{78}$ SVBRDF from a single view using 1 DOF-moving linear light 79 source and a set of known BRDF samples recorded simultane80 ously with the sample [27]. Recently, sparse SVBRDF mea${ }_{81}$ surement and reconstruction have been used based on the mea82 surement of several images of known geometry illuminated by вз a circularly polarized light [8]. Although this method requires 84 the capture of only four sample images, its usage is limited to 85 flat and isotropic measurements and it requires a complex mea${ }_{86}$ surement setup.

${ }_{87}$ View and illumination dependent data interpolation is of88 ten performed when sparse images of known geometry and ils9 lumination direction are recorded. Reflectance data collected ${ }_{90}$ from such images can be interpolated either by a parametric 91 reflectance model [17], or in the form of isotropic BRDFs in${ }_{92}$ terpolated by means of three-dimensional radial basis functions ${ }_{93}$ [32] in achieving a reconstruction of SVBRDF. Alternatively, a ${ }_{94} 4 \mathrm{D}$ BRDF can be decomposed into simpler 1D and 2D compo${ }_{95}$ nents having physical meaning, to allow parametric editing of ${ }_{96}$ visual properties [16].

However, to the best of our knowledge, no measurement ${ }_{98}$ technique yet exists enabling rapid capture of anisotropic ABRDF 99 using an consumer camera and light. Proposed is a method 100 for fast, non-restricted anisotropic ABRDF space reconstruc101 tion from extremely sparse samples that can be measured in a 102 few seconds by continual movement of the camera and light. ${ }_{103}$ Contrary to parametric BRDF models [24] or other simplified 104 solutions (see Fig. 2), this method is capable of correctly recon105 structing non-reciprocal, non-energy-conserving ABRDF data.

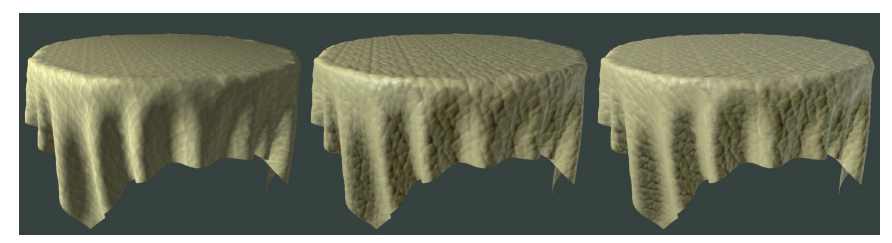

Figure 2: A comparison of renderings based on a single texture modulated by an analytical BRDF model [14] (left), reference BTF measurements using 6561 images (middle), and the proposed sparse data selection and reconstruction using 168 images (right).

106

Although the principle of the method has been outlined in 108 [7], this paper provides additional thorough reasoning of the 109 method's functionality. In addition, an introduction to a novel 110 interpolation technique for missing elevations, results of real 111 appearance acquisition, and the method's application on sparse 112 reconstruction of BTF datasets are included.

\section{${ }_{113}$ 3. The Proposed Reconstruction Method}

A robust and sparse acquisition of general view and illu115 mination dependent appearances is a tricky task. While a po116 sition of specular highlights can be expected near the mirror 117 reflection, the location of anisotropic highlights is unknown. It 
118 depends on the local macro-geometry as well as on the micro119 geometry of the measured surface. In our work we look for a 120 very sparse set of illumination/view measurement points. They ${ }_{121}$ should allow a visually tolerable reconstruction of material re122 flectance, as well as a quick measurement of the sparse dataset ${ }_{123}$ using simple inexpensive hardware. There would be no need to ${ }_{124}$ preprocess the measured material sample or remove it from its 125 environment.

126 Standard angularly uniform or even adaptive sampling strate${ }_{127}$ gies require many samples to preserve high frequencies in the ${ }_{128}$ data. On the other hand, employing analytical BRDF models 129 imposes restrictions on data reciprocity and requires lengthy 130 fitting, etc. Therefore, we analyzed a typical ABRDF and em${ }_{131}$ ployed this knowledge to capture and reconstruct its behavior 132 using a small set of measurements. This analysis has shown that ${ }_{133}$ it is most effective to place samples perpendicularly to specular ${ }_{134}$ highlights in a subspace of view/illumination azimuths. This 135 is done in such a way that the samples form slices in the sub${ }_{136}$ space and can be easily measured by horizontal movement of ${ }_{137}$ the light/camera around the measured sample. As the appear${ }_{138}$ ance of the azimuthal subspaces often depends on elevation an${ }_{139}$ gles, to create a more precise approximation we suggest sam140 pling four combinations of view/illumination elevations.

${ }_{141}$ A principle of the proposed method [7] is explained in Fig. 3.

First, the material's ABRDF (Fig. 3-a) is sparsely measured in

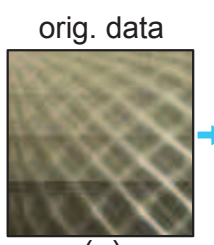

(a)

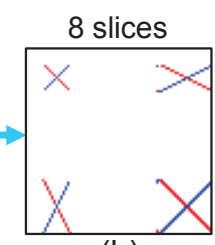

(b)

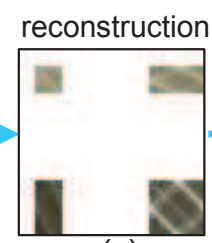

(c)

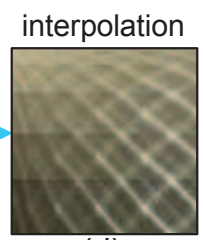

(d)
Figure 3: Example of ABRDF reconstruction: (a) original, (b) sparse-sampling using 8 slices, (c) reconstructions of elevations where the slices were measured, (d) missing data interpolation.

142

143 four subspaces by means of eight slices (Fig. 3-b), then the 144 missing values in these subspaces are reconstructed from the 145 values of the slices (Fig. 3-c), and lastly the remaining values at 146 non-measured elevations are interpolated (Fig. 3-d).

\section{3.1. Acquisition of Slices}

148

Because one of our major concerns is the simplicity and 149 speed of the acquisition process, we suggest taking samples by 150 the continuous movement of light/camera around the sample at ${ }_{151}$ fixed elevations. By doing so, samples can be taken at an ar152 bitrary density, limited only by camera movement speed and ${ }_{153}$ frame-rate. Each subspace of azimuthal angles $\varphi_{i} / \varphi_{v}$ is sam154 pled by means of two perpendicular slices (see Fig. 5-a), which 155 differ in the direction of mutual movement of camera and light. 156 In principle, the slices are, for a majority of the materials, or157 thogonal to their most prominent features: a specular reflection 158 and an anisotropic reflection (see Fig. 4). These features are of159 ten constant in the direction perpendicular to the slices and thus 160 can be effectively represented by their marginal values.

The slice aligned with the direction of the specular high162 lights is called axial slice $s_{A}$ (red), i.e., $\varphi_{v}-\varphi_{i}=\alpha$ holds
${ }_{163}$ for azimuthal angles. The axial slice records the material's 164 anisotropic properties (mutual positions of the light and cam165 era are fixed while the sample rotates (see Fig. 14-left)), i.e., 166 its value is almost a constant for near-isotropic samples.

The slice perpendicular to the highlights is called diagonal ${ }_{168}$ slice $s_{D}$ (blue), i.e., $\varphi_{i}+\varphi_{v}=2 \pi$ holds for azimuthal angles. The ${ }_{169}$ diagonal slice captures the shape of the specular peaks (light 170 and camera travel in mutually opposite directions over the sam${ }_{71}$ ple (see Fig. 14-right)).
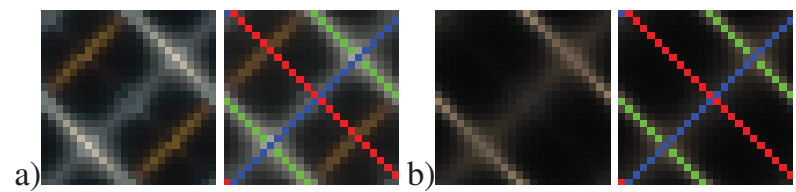

Figure 4: Examples of ABRDF toroidal subspaces for derivation of optimal placement of the axial slices (green and red alternatives).

We focused first on analysis of the ABRDF subspace hav173 ing the highest elevations, in which the illumination and view 174 dependent effects are the most pronounced. See in the first row 175 of Fig. 7. The azimuthal difference of light and camera during 176 axial slice measurement $-\alpha$ influences the placement of slices 177 in the ABRDF toroidal subspace; therefore, we analyzed opti178 mal placement of axial and diagonal slices across a number of ${ }_{179}$ ABRDFs. The study has shown that while the placement of a 180 diagonal slice can be arbitrary, the highest variance along axial ${ }_{181}$ slices is achieved near the specular highlight. Consequently, 182 this is - most likely - the best placement of the axial slice ${ }_{183} \alpha=180^{\circ}$ (green dots in Fig. 4). However, such a placement 184 might omit vital color/luminance information in some parts of 185 the subspace. For example, it would completely miss yellow 186 anisotropic features as in Fig. 4-a or dark parts as in Fig. 4-b. ${ }_{187}$ Therefore, we used the slice with the second highest variance ${ }_{188} \alpha=15^{\circ}$ (red dots in Fig. 4) to eliminate occlusion of the camera 189 with the light and capture most visual features of the ABRDF 190 subspace (see the shift of the red axial slice from image diago191 nal in Fig. 5-a).

192 For experimental purposes the slices can be taken from the 193 measured ABRDF (Fig. 5-a) as

$$
\begin{aligned}
s_{A, \theta_{i} \theta_{v}}\left(\varphi_{i}\right) & =\operatorname{ABRDF}\left(\theta_{i}, \theta_{v}, \varphi_{i}, \varphi_{v}=\varphi_{i}+\alpha\right), \\
s_{D, \theta_{i} \theta_{v}}\left(\varphi_{v}\right) & =\operatorname{ABRDF}\left(\theta_{i}, \theta_{v}, \varphi_{i}=2 \pi-\varphi_{v}, \varphi_{v}\right) .
\end{aligned}
$$

\section{3.2. Reconstruction from slices}

195 ABRDF toroidal subspace reconstruction is performed for ${ }_{196}$ elevation angles at which the slices were captured. It can be 197 explained as a combination of two slices (i.e., sets of marginal 198 values) as shown in Fig. 5. The reconstruction of point $199 \widehat{A B R D F}\left(\theta_{i}, \theta_{v}, \varphi_{i}, \varphi_{v}\right)$ in ABRDF subspace starts with combin200 ing contributions of the $s_{A}$ and $s_{D}$ slices. We tested their sum 201 and product; however, the latter improperly enhanced the loca202 tions at intersections of the specular and anisotropic highlights 203 as shown in Fig. 6-b. Note that the sum of slice contributions 204 (see Fig. 6-c) preserves specular highlights, which are less af205 fected by the anisotropic highlights. Therefore, we finally used 206 the sum of slices in our reconstruction procedure:

$$
v_{\theta_{i} \theta_{v}}\left(\varphi_{i}, \varphi_{v}\right)=s_{A, \theta_{i} \theta_{v}}\left(\varphi_{i, R}\right)+s_{D, \theta_{i} \theta_{v}}\left(\varphi_{v, R}\right),
$$




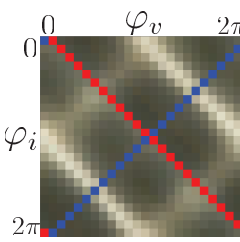

(a)

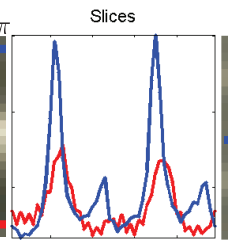

(b)
Reconstruction $\pi / 4$

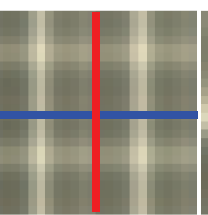

(c)
Reconstruction final

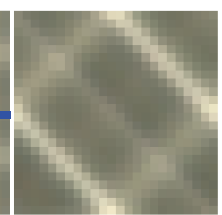

(d)
Figure 5: Reconstruction of a toroidal ABRDF subset from two slices at fixed elevations: (a) reference data with slice placements, (b) data profiles in the slices, (c) reconstruction from slices $\left(\frac{\pi}{4}\right.$ rotated), (d) final reconstruction.

$$
\left[\begin{array}{l}
\varphi_{i, R} \\
\varphi_{v, R}
\end{array}\right]=\left[\begin{array}{cc}
\cos (\pi / 4) & -\sin (\pi / 4) \\
\sin (\pi / 4) & \cos (\pi / 4)
\end{array}\right]\left[\begin{array}{l}
\varphi_{i} \\
\varphi_{v}
\end{array}\right]
$$

(a)

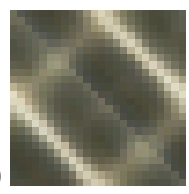

(b)

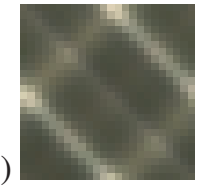

$14.0 / 20.9 / 21.7$ (c)

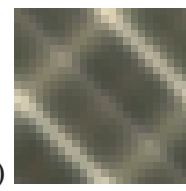

$9.6 / 12.2 / 26.5$
Figure 6: Original subspace (a), and its reconstructions using product of slices (b) and sum of slices (c). Below are the difference values in: CIE $\Delta$ E / RMSE / PSNR[dB]

207 Note that the original azimuths $\varphi_{i}, \varphi_{v}$ had to be rotated for $208 \pi / 4$ (Fig. 5-c) to account for the slant of slices with respect to 209 the $\varphi_{i}, \varphi_{v}$ coordinate system (Fig. 5-a). Finally, the summed 210 value $v$ is mapped to a dynamic range of original slices

$$
\begin{aligned}
& 211 \quad \widehat{A B R D F}\left(\theta_{i}, \theta_{v}, \varphi_{i}, \varphi_{v}\right)=v_{\theta_{i} \theta_{v}}\left(\varphi_{i}, \varphi_{v}\right) \cdot(M-m)+m, \\
& { }_{212} \quad m=\min \left(s_{A, \theta_{i} \theta_{v}} \cup s_{D, \theta_{i} \theta_{v}}\right) \quad M=\max \left(s_{A, \theta_{i} \theta_{v}} \cup s_{D, \theta_{i} \theta_{v}}\right)
\end{aligned}
$$

213 Since the axial slice always has a constant value for isotropic 214 samples, the slices do not have to be combined and reconstruc215 tion can be performed using the diagonal slice alone as

216

$$
\widehat{A B R D F}\left(\theta_{i}, \theta_{v}, \varphi_{i}, \varphi_{v}\right)=s_{D, \theta_{i} \theta_{v}}\left(\varphi_{v, R}\right)
$$

217 Fig. 7 shows a reconstruction of anisotropic ABRDF sub218 space at elevations $\theta_{i} / \theta_{v}=75^{\circ} / 75^{\circ}$ (the second row) from two ${ }_{219}$ slices (the third row) and prove the ability of the proposed approach to represent a variety of anisotropic materials.

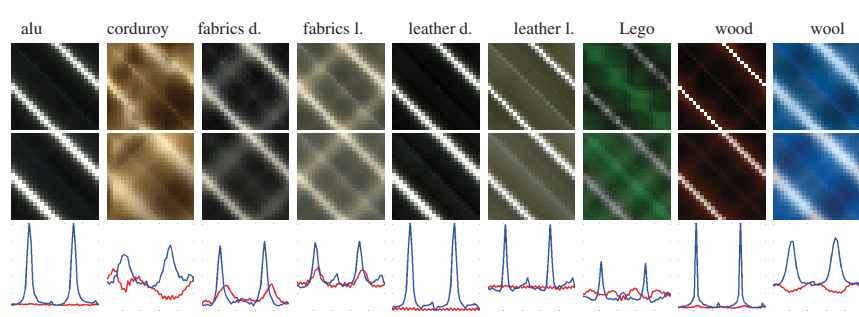

Figure 7: Comparison of the material's ABRDF toroidal subspace at elevation $75^{\circ}$ (the first row), with its reconstruction (the second row) from the axial (red) 220 and diagonal (blue) slices (the third row).

\section{3.3. Interpolation of missing values}

222 At this point, sparse acquisition and reconstruction of the 223 ABRDF subspace has been explained. However, the selection
224 of elevations at which the slices are measured significantly in225 fluences the final ABRDF reconstruction. Therefore, we per226 formed an experiment with two measured ABRDFs (isotropic 227 specular and diffuse anisotropic material) in order to find the 228 proper combination of two elevations at which the four sub229 spaces should be measured. We tested six different combina230 tions of elevations. Only samples from these illumination/view 231 elevations were used for the entire ABRDF interpolation us232 ing radial basis functions [26]. The average RMSE differences 233 between ground-truth ABRDF data and interpolation shown in ${ }_{234}$ Fig. 8 suggest that the combination of $\theta=45^{\circ} / 75^{\circ}$ provides the lowest reconstruction error. Therefore, we have chosen

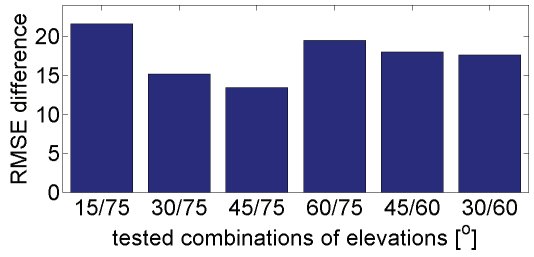

Figure 8: ABRDF reconstruction error (RMSE) for different elevation combinations used for selection of four measured subspaces.

235

${ }_{236}$ the highest elevation angles $\theta_{i}=\theta_{v}=75^{\circ}$, where the specu${ }_{237}$ lar reflections are the most intensive (see first row of Fig. 12). 238 The lower elevation angles were decreased to $\theta_{i}=\theta_{v}=30^{\circ}$ 239 (the second best choice from Fig. 8) for better representation 240 of material appearance at orthogonal viewing and illumination 241 directions, which are the most visually salient. More than these 242 four subspaces can be used at the expense of more camera/light 243 elevations; however, this would increase the number of sam${ }_{244}$ ples and the complexity of their measurement. Finally the four 245 subspaces at the following elevations were sampled: $\theta_{i} / \theta_{v}=$ ${ }_{246} 30^{\circ} / 30^{\circ}, 75^{\circ} / 75^{\circ}, 30^{\circ} / 75^{\circ}, 75^{\circ} / 30^{\circ}$,).

247 However, data for the remaining subspaces are still unknown 248 and have to be estimated. The BRDF parametric models, e.g., 249 [23], cannot be used to solve this problem because they impose 250 restrictions on data properties (reciprocity, energy conservation, 251 etc.), require many more samples or a different distribution of 252 samples, and lengthy fitting. They also depend on initial values. ${ }_{253}$ We tried to fit measured samples using the anisotropic paramet254 ric BRDF model [14]. Due to a low number of samples and 255 their distribution we were unable to find a stable parameter fit 256 for most of the tested ABRDFs. Moreover, these models are 257 not designed to handle non-reciprocal ABRDF data. Therefore, 258 we tested the following two interpolation approaches:

259 Method A - In the first one, the interpolation was performed 260 by means of the four-dimensional radial basis functions [26] 261 computed separately in each color channel. We tested several 262 parameterizations of illumination and viewing directions, e.g., 26з $\left[\theta_{i}, \varphi_{i}, \theta_{v}, \varphi_{v}\right],\left[\theta_{h}, \varphi_{h}, \theta_{d}, \varphi_{d}\right]$ from [28], and finally used param264 eterization according to [11], applied to both illumination and 265 view directions $\left[\alpha_{i}, \beta_{i}, \alpha_{v}, \beta_{v}\right]$. This parameterization has shown 266 the lowest reconstruction error due to alignment of specular 267 highlights $0^{\circ}$ value of angle $\beta_{i}$.

${ }_{268}$ Method B - Due to relatively high computational demands of ${ }_{269}$ Method A, we developed a faster hybrid linear interpolation 270 constrained by a reflectance model. This interpolation consists 
271 of two steps shown in Fig. 9. First we interpolate data at dif272 ferent viewing and constant illumination elevations. Then the remaining illumination elevations are filled. In each interpo-

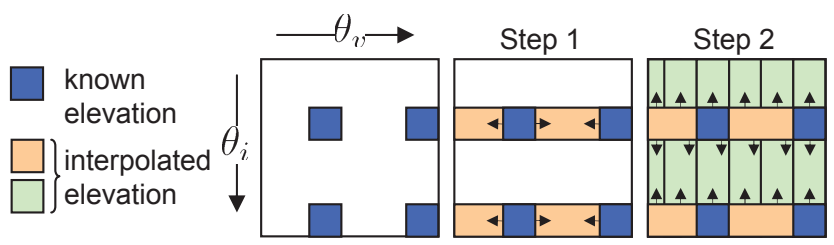

Figure 9: Steps of the interpolation approach $A$.

274 lation step, the average ABRDF value for each unknown ele275 vation was approximated by fitting a simplified monospectral 276 one-lobe Lafortune model [15] with parameters $k, \alpha$ to known 277 slice values

278

$$
f_{r}(k, \alpha)=k\left(\cos \theta_{i} \cdot \cos \theta_{v}\right)^{\alpha} .
$$

279 Initialization of $k, \alpha$ was constant during all experiments. Val280 ues obtained from the model at elevations $\theta$ are scaled by the 281 mean values of the slices and then used for obtaining inter282 polation weights. These weights are then applied for a linear 283 interpolation of missing elevations from slice values at known 284 elevations as shown in Fig. 10. Elevations lower than $30^{\circ}$ are 285 extrapolated using the scaled model's (6) predictions. This pro286 cedure is performed over all azimuthal directions as shown in Fig. 9.

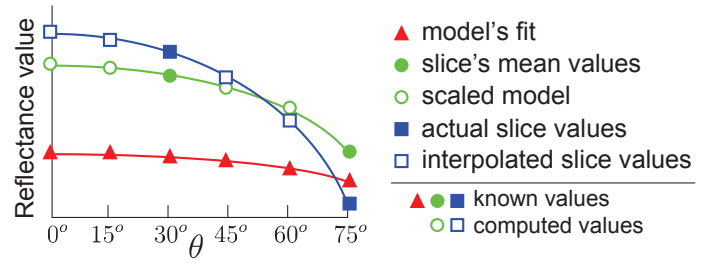

Figure 10: An interpolation of non-measured elevation values.

287

288

289 ally intensive than Method B and provides better results in most 290 cases. While Method A allows arbitrarily dense sampling, even 291 for originally unmeasured azimuthal directions, Method B re292 constructs ABRDF data in their original azimuthal sampling. ${ }_{293}$ The results in Fig. 11 show the major visual differences be294 tween both proposed interpolation methods in two illumination 295 environments.

296 We also tested modification of this step-wise interpolation 297 of subspaces (Fig. 9) using a displacement interpolation (de298 noted as BD) method [2]. Compared to the weighted linear 299 interpolation (Method B), its principle is based on solving the 300 generalized mass transport optimization problem. As this method 301 cannot extrapolate, the low elevation areas were reconstructed 302 using Method B. Method BD gives better results than Method ${ }_{303} \mathrm{~B}$ and comparable results to Method $\mathrm{A}$. The whole subspace in304 terpolation is about thirty times slower than Method A, while 305 when view or illumination direction is fixed, i.e., individual 306 lines in subspaces are interpolated separately, its speed com307 pares to Method A. For this reason we have not used Method ${ }_{308} \mathrm{BD}$ further in the paper.
Although both global (A) and local (B) interpolation ap310 proaches provide similar visual quality (see more discussion 311 in Section 4), we believe their performance can be further im312 proved, e.g., using estimated height-map as an interpolation 313 constraint.

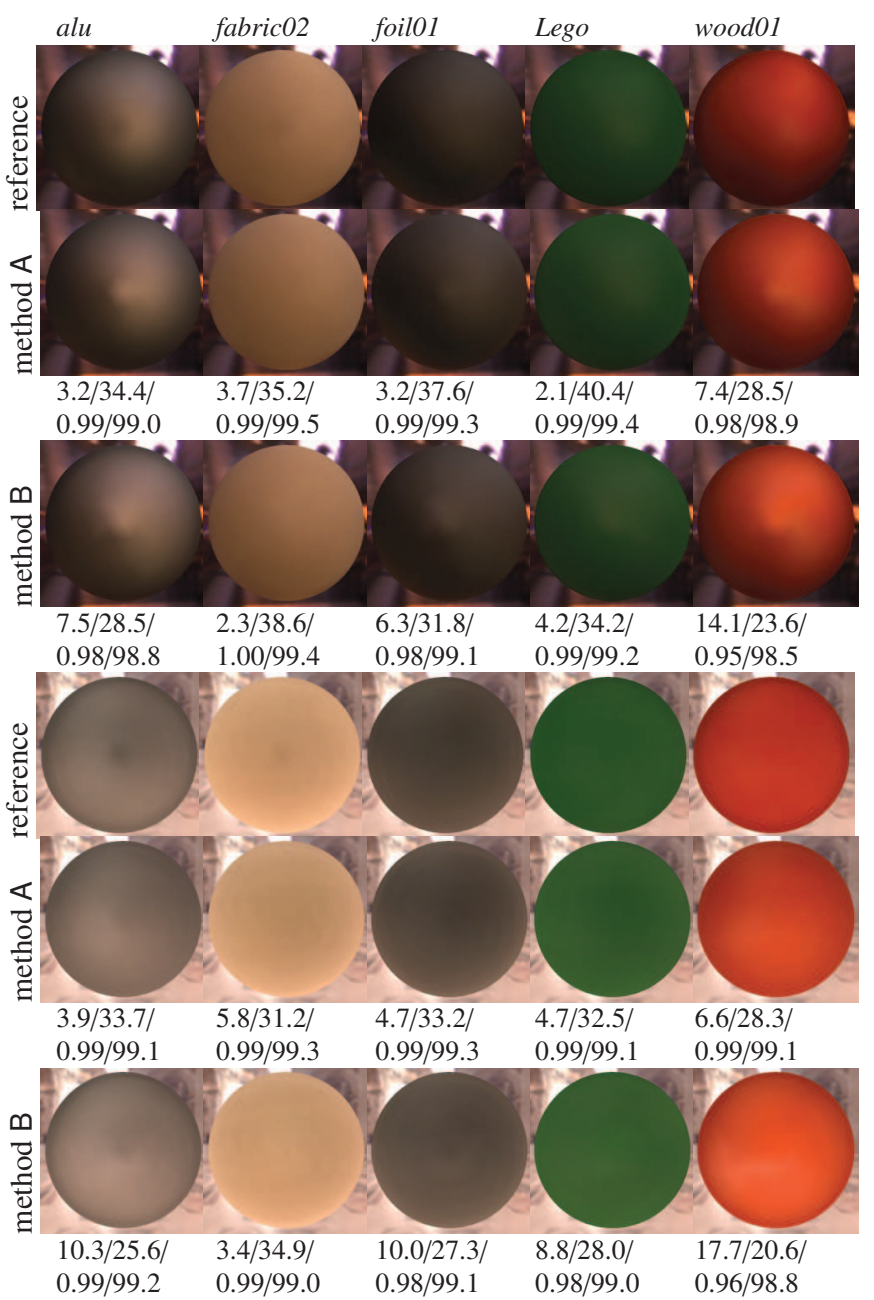

Figure 11: A comparison of the interpolation methods performance in grace and st.peters illumination environments [5]. Below are the CIE $\triangle \mathrm{E} / \mathrm{PSNR}[\mathrm{dB}] /$ SSIM / VDP2 difference values.

\section{${ }_{314}$ 4. Results of Simulated Measurement}

In this section we show results of sparse reconstruction ex316 periments performed on isotropic BRDF and anisotropic ABRDF 317 data. The data served as a source of sparse sampling and were 318 simultaneously used for evaluating reconstruction quality of the 319 method.

320 Generally, the angular resolution used in all experiments in 321 this paper was 81 view $\times 81$ illumination directions (6561 val322 ues) [29] distributed uniformly over the hemisphere (Fig. 1) To 323 sample this resolution in the slices of the proposed method we 324 need only 168 samples to obtain information sufficient for data 325 reconstruction.

${ }_{326}$ In the first experiment, we tested our method on reconstruc327 tion of 55 isotropic BRDF samples (resampled to $81 \times 81$ di328 rections) from the MERL BRDF database [19]. The advantage 329 of isotropic reconstruction is that only four diagonal slices $s_{D}$ 
${ }_{330}$ have to be obtained (in our case 84 samples instead of the 168 ${ }_{331}$ needed for anisotropic data). Mean reconstruction errors of all ${ }_{332} 55$ BRDFs (8bits/channel) were: $\mathrm{CIE} \Delta \mathrm{E}=9.1, \mathrm{RMSE}=15.7$, $3 з$ and $\mathrm{PSNR}=24.9$ [7].

${ }_{334}$ In the second experiment, ten BTF samples (nine from Bonn ${ }_{335}$ University BTF database $^{1}$ and one from Volumetric Surface ${ }_{336}$ Texture Database $^{2}$ ) were used (aluminum profile, corduroy, dark 337 and light fabrics, dark and light leatherettes, lacquered wood, 338 knitted wool, upholstery fabric Proposte, and Lego). These ma$3 з 9$ terials, due to their rough structure and often non-opaque prop340 erties, exhibit anisotropic effects of occlusions, masking, sub${ }_{341}$ surface scattering and therefore represent a challenging dataset 342 to test the proposed method. All BTF pixels were averaged to 343 obtain the average ABRDF of the material (first row of Fig. 12). ${ }_{344}$ The results of the complete reconstruction of original ABRDFs 345 from 168 sparse samples are shown in Fig. 12. Together with 346 difference images $(10 \times$ scaled $)$ and reconstruction errors in terms 347 of CIE $\triangle E$, RMSE, PSNR, these results show that even a very 348 sparse set of measured values can provide promising recon349 struction of such challenging anisotropic datasets. Although on 350 average both interpolation approaches performed similarly, the ${ }_{351}$ difference images in Fig. 12 show that the global interpolation 352 Method (A) estimated incorrect values for elevations between 353 the two sampled elevation values $\left(30^{\circ}\right.$ and $\left.75^{\circ}\right)$. On the other 354 hand, Method (B) gives, due to a lack of global knowledge, the 355 worst estimation for extrapolated elevations. That is, elevations 356 smaller than $30^{\circ}$ as represented by the first few rows/columns 357 in the images. Finally, the displacement interpolation - Method ${ }_{358}$ BD scored similarly to Method A. The reconstruction and inter359 polation of a single ABRDF from 168 samples take $\approx 1$ second 360 using interpolation Method $A, \approx 0.3$ second using Method $B$, 361 and $\approx 1$ second using Method BD on Intel Xeon $2.7 \mathrm{GHz}$ (using 3623 cores).

To validate the contribution of our method, we compared 364 its reconstruction performance using 168 samples with the uni365 form sampling of a similar samples count. For this purpose, 366 hemispheres of illumination and viewing directions were sam367 pled by means of $13 \times 13$ samples, producing a total of 169 sam${ }_{368}$ ples. Then the missing values in the ABRDF space were inter369 polated from these sparse samples by means of four-dimensional 370 radial basis functions [26] (Method A). The interpolation was 371 computed separately in each color channel, and $0 \approx 2 \pi$ discon372 tinuity has been avoided using the onion parameterization of 373 illumination and view directions [11]. Comparison of ten inter${ }_{374}$ polated ABRDFs (see Fig. 13) has shown that the proposed re375 construction method has a better performance than the interpo376 lation from uniform samples, mainly near specular highlights, 377 as confirmed by the objective criterion values shown below the 378 reconstructions. On average, the proposed reconstruction pro379 vides 1.4 and 3.2 lower $\triangle \mathrm{E} / \mathrm{RMSE}$ values and 1.8 higher PSNR 380 value across ten tested ABRDFs. Moreover, the data acquisi${ }_{381}$ tion process using our method is considerably faster and less 382 demanding on hardware as shown in Section 6.

\footnotetext{
${ }^{1}$ http://btf.cs.uni-bonn.de/

${ }^{2}$ http://vision.ucsd.edu/kriegman-grp/research/vst/
}

\section{${ }_{383}$ 5. Limitations}

The limitations of the proposed method are threefold. First, 385 since the method restores reflectance at given elevations only 386 from two orthogonal slices, it cannot reliably capture features 387 that are not orthogonal to the slices (see second example of 388 corduroy in Fig. 7). It must also be noted that the proposed 389 slices represent a very sparse sampling of the azimuthal sub390 space and as such, can omit some reflectance features, resulting 391 in a slightly different color/brightness appearance of the recon392 structed data. To avoid this problem, the azimuthal subspace 393 can be sampled by additional slices at the cost of slightly longer 394 acquisition times. Second, the interpolation step of the algo395 rithm expects monotonicity of reflectance values across differ396 ent illumination and view elevations. However, this condition 397 is rarely invalid and no such behavior was experienced with any 398 of the tested materials. The method's accuracy can be further 399 improved in this respect by taking more slices at different eleva400 tions. Finally, highlights of extremely specular samples are not 401 always represented accurately enough (see Fig. 19) mainly due 402 to an insufficient angular sampling of azimuthal angles (step ${ }_{403} 15^{\circ}$ ) in original datasets used in the experiments. Note that the 404 sampling density along specular highlights in diagonal slices 405 can be arbitrarily increased to provide a better match of specu406 lar highlights of a high-dynamic-range within the model with407 out increasing the measurement time.

408 Note that the proposed method does not fit any analytical 409 model to the measured data and as such it is sensitive to noise 410 in the measurement process. However, since the measurement ${ }_{411}$ procedure is fast and simple, this noise can be effectively sup${ }_{412}$ pressed by measuring the slices several times and computing ${ }_{413}$ the measurements' median values.

\section{6. Sparse ABRDF Data Measurement}

415 This section describes a practical experiment of capturing ${ }_{416}$ sparse ABRDF samples using a consumer camera and a LED 417 point-light source and is followed by a complete ABRDF re${ }_{418}$ construction from such measurements.

Mutual movement of arms with camera and light with re420 spect to the sample being measured is controlled manually as is shown in Fig. 14. The axial slice $s_{A}$ data (left) are measured
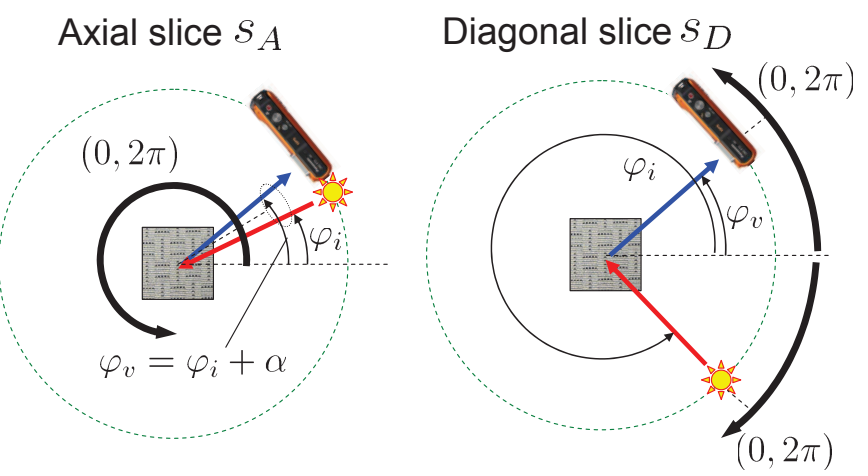

Figure 14: The proposed ABRDF measurement setup at fixed elevation angles $\theta_{i} / \theta_{v}$

422 using rotation of the fixed light and sensor around the sample, 

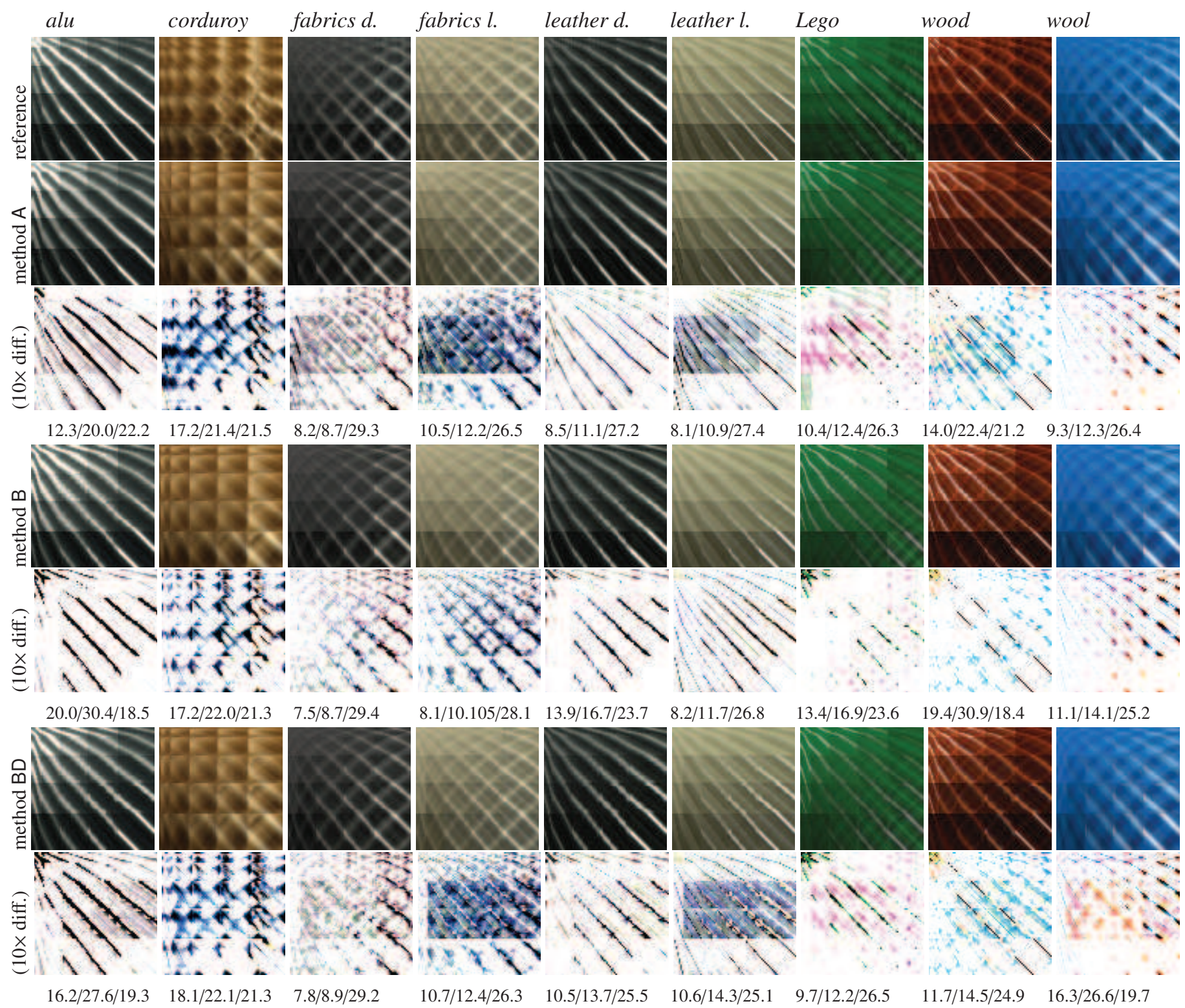

Figure 12: Comparison of the material's BRDF (the first row), and interpolation of missing values by means of method A (the second row), method B (the third row), and its modification method BD (the fourth row) respectively. Below are $10 \times$ difference images and global difference values in CIE $\triangle E$ / RMSE / PSNR[dB].

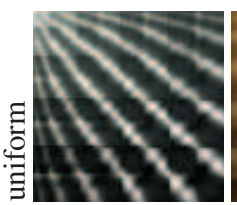

17.1/27.3/19.4

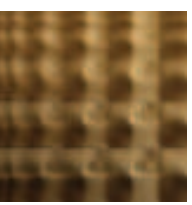

$9.8 / 13.7 / 25.4$

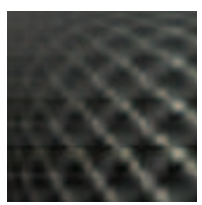

9.4/13.4/25.6

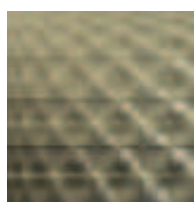

9.8/13.4/25.6

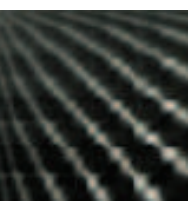

$13.6 / 22.2 / 21.3$

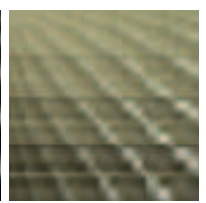

$11.4 / 18.0 / 23.0$

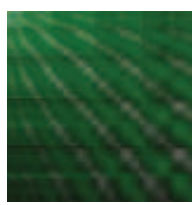

24.1/20.6/21.9
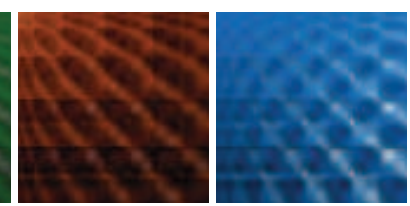

Figure 13: The performance of interpolation from 169 sparse uniform samples (13 samples per hemisphere). Below are the difference values in: CIE $\Delta \mathrm{E} / \mathrm{RMSE} / \mathrm{PSNR}[\mathrm{dB}]$

${ }_{423}$ while the diagonal slice $s_{D}$ data (right) are obtained by mutu- ${ }_{431}$ structed a frame with two arms using a Merkur toy ${ }^{3}$ construc${ }_{424}$ ally opposite movements of the light and sensor in respect to 432 tion set shown in Fig. 15-b. During its movement, the camera ${ }_{425}$ the sample. Both the camera and light travel full circle around ${ }_{433}$ records the material sample appearance as a video sequence at ${ }_{426}$ the sample and return to the initial position. 434 a resolution of $1280 \times 720$ pixels, and the elevation angles of ${ }_{427}$ Our acquisition setup consisted of the Panasonic camera ${ }_{435}$ the camera and light are kept constant using the setup Fig. 15${ }_{428}$ Lumix DMC-FT3 and light using high-power LED Cree XLamp ${ }_{436} \mathrm{~b}$. Both $s_{A}$ and $s_{D}$ slices are recorded for two different ele${ }_{429} \mathrm{XM}-\mathrm{L}$ with $20^{\circ}$ frosted optics (Fig. 15-a). To achieve the re430 quired synchronous movement of light and camera, we con-

${ }^{3} \mathrm{http}: / /$ www.merkur.cz/ 


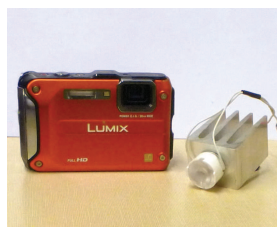

(a)

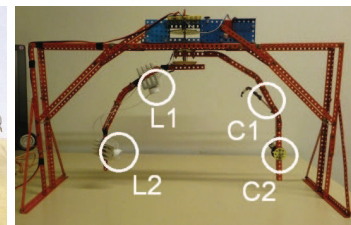

(b)

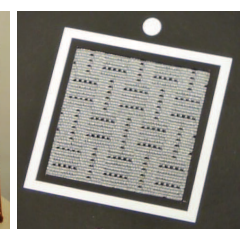

(c)
Figure 15: Data acquisition equipment (a) with its fixating frame (b), and measured sample with registration borders for calibration (c).

${ }_{437}$ vations of the camera $(\mathrm{C} 1, \mathrm{C} 2)$ and light $(\mathrm{L} 1, \mathrm{~L} 2)$; therefore, 438 eight slices are measured approximately at elevations $\theta_{i} / \theta_{v}=$ ${ }_{439}\left[30^{\circ} / 30^{\circ}, 30^{\circ} / 75^{\circ}, 75^{\circ} / 30^{\circ}, 75^{\circ} / 75^{\circ}\right]$ as shown in Fig. 3-b. Record 440 ing of the slices took less than 10 minutes. From each of the ${ }_{441}$ eight video sequences, 24 frames were extracted corresponding ${ }_{442}$ to sampling of azimuthal angles $\varphi_{i} / \varphi_{v}$ every $15^{\circ}$. This resulted 443 in a total of 192 samples being obtained. The number differs ${ }_{444}$ from 168 samples used in the reconstructions in Section 4, be445 cause this time all elevations were covered by the same num446 ber of samples. The effective number of samples is always ${ }_{447}$ slightly lower than 192, as some of the frames are removed ${ }_{448}$ due to occlusion of the material by the arm with light. Note ${ }_{449}$ that the method's principle allows adaptive density of the sam${ }_{450}$ ples (frames) along the slices to also record extremely narrow ${ }_{451}$ specular highlights.

${ }_{452}$ Three anisotropic fabric materials $(30 \times 30 \mathrm{~mm})$ were used ${ }_{453}$ as test samples, as shown in Fig. 16. A white border was at454 tached around the sample to help detect camera orientation in 455 respect to the sample coordinate space and for sample registra456 tion Fig. 15-c). Because of this, we first calibrated the camera 457 [31]. Unfortunately, the used low-end camera adapts its expo458 sure depending on the amount of light coming from the scene. ${ }_{459}$ On the other hand, this feature enables us to capture as much ${ }_{460}$ information as possible, even using a limited dynamic range of ${ }_{461}$ the camera's sensor (8bits/color). Since the information about ${ }_{462}$ exposure throughout the video sequence could not be retrieved ${ }_{463}$ from an EXIF header as is possible for still photos, we used the 464 reference BRDF data of dark material surrounding the sample 465 to compensate for exposure of each image. That is, we com${ }_{466}$ pensated color values of the sample using the black part of the ${ }_{467}$ sample holder (near the white borders as shown in Fig. 15-c) ${ }_{468}$ and its reference measurements.

469 Subsequent processing was then performed for each image. ${ }_{470}$ Camera viewing angles $\theta_{v} / \varphi_{v}$ were obtained from camera ex${ }_{471}$ trinsic parameters, given the known camera calibration and cor472 ner points of the white borders. Coordinates of these points 473 were obtained from the image registration based on the camera 474 calibration. When the viewing angles were known, the illumi475 nation azimuth angle was computed as: $\varphi_{i}=\varphi_{v}-\alpha$ for the axial ${ }_{476}$ slice $s_{A}$, and $\varphi_{i}=2 \pi-\varphi_{\nu}$ for the diagonal slice $s_{D}$. The elevation 477 angles $\theta_{i}$ were estimated from the slant of the light during mea478 surement. Finally, the slice's ABRDF value from each image 479 was obtained as the average of RGB values near the sample's 480 center, and colorimetrically calibrated. The non-optimized data ${ }_{481}$ processing described above took approximately 10 minutes to ${ }_{482}$ perform over all selected images. The reference ABRDF mea483 surements of the black target and materials are obtained from
${ }_{484}$ the UTIA BTF database ${ }^{4}$ and have the same angular resolution 485 as BTF Database Bonn [29].

486 When all of the selected images were processed in this way ${ }_{487}$ and data for all eight slices were obtained, the ABRDF space re${ }_{488}$ construction described in Sections 3.2 and 3.3 was performed. ${ }_{489}$ Figure 17 compares reference ABRDF measurements of the 490 material (a) with their reconstruction from the 192 sparse ref${ }_{491}$ erence samples using Method B (b), and with a reconstruction 492 using 192 sparse measurements obtained by the proposed setup 493 and interpolation Method B (c). The last column (d) of Fig494 ure 17 compares our method with a uniform sampling using 196 495 samples $\left(14^{2}\right)$, while the remaining samples are interpolated us496 ing Method A (compare with column (b)). Note that, while the ${ }_{497}$ visual performance of the uniform sampling might look simi498 lar, the complexity of its measurement is considerably higher in 499 comparison with the proposed measurement approach.

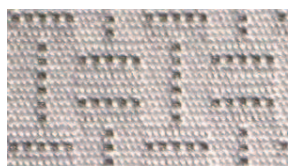

fabric01

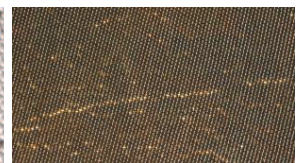

fabric02

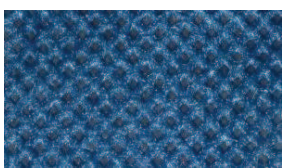

fabric03
Figure 16: Three anisotropic fabric samples whose ABRDFs were measured using the proposed setup.

Finally we took photographs of the fabric 02 and fabric 03 501 materials attached on a cylinder (a) and compared them with 502 renderings on a cylinder using their reference BRDFs (b) and ${ }_{503}$ BRDFs captured by the proposed setup (c). The results for dif504 ferent illumination conditions are shown in Fig. 18 and con505 firm that even the proposed approximate measurement setup 506 can record BRDFs with reasonable accuracy, in comparison to 507 the reference measurements.

The reconstruction results from our preliminary measure509 ments (Figure 17-c) are encouraging and we believe that they 510 convey the idea of ABRDF capturing speed and simplicity with${ }_{511}$ out the need for dedicated and thus costly devices.

A notable advantage of our setup and the proposed sampling ${ }_{513}$ pattern is its ability to quickly measure any flat samples without 514 needing to extract them from their environment, and thus it can 515 be used for fast and inexpensive measurements of such samples 516 as human skin and precious cultural heritage objects.

\section{Experimental BTF Reconstruction}

As the acquisition and reconstruction of spatially-varying 519 datasets is a straightforward extension of the proposed sparse 520 sampling and reconstruction method, we tested the method's ${ }_{521}$ performance on ten BTF samples of angular resolution $81 \times$ ${ }_{522} 81=6561$ images as described in Section 4. Only 168 im${ }_{523}$ ages (corresponding to the eight data slices) were selected from 524 the BTF samples and used for pixel-wise reconstruction of the 525 remaining images using the proposed method.

\footnotetext{
${ }^{4}$ http://btf.utia.cas.cz
} 


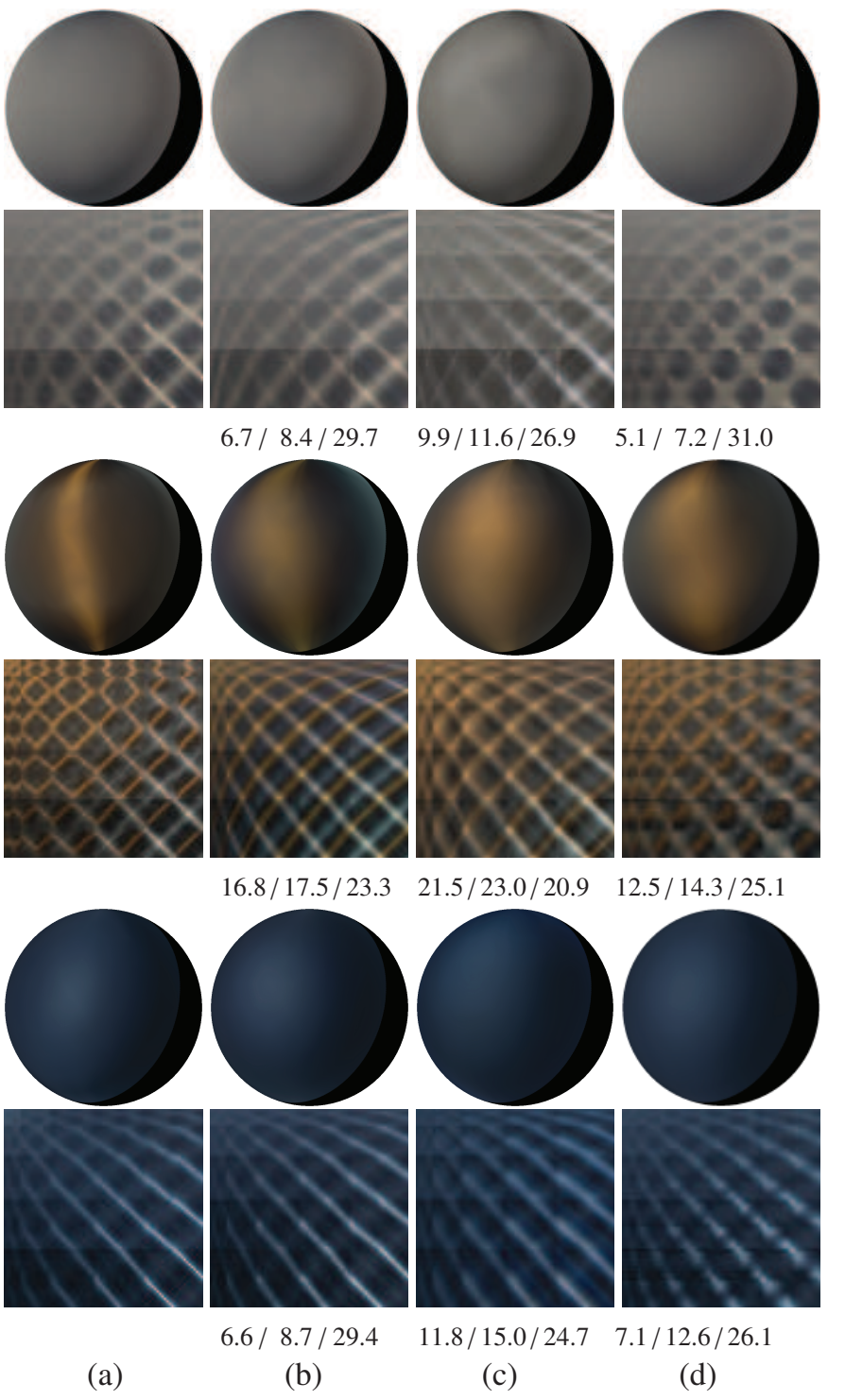

Figure 17: ABRDF reference measurement (a), compared to reconstruction from 168 sparse reference measurements only using Method B (b), and reconstruction from the proposed measurement procedure using 192 samples and interpolation Method B (c), uniform interpolation using 196 samples (d). Below are the difference values in: CIE $\Delta \mathrm{E} / \mathrm{RMSE} / \mathrm{PSNR}[\mathrm{dB}]$.

\section{7.1. Results}

527 Renderings of the original data with results of the proposed 528 reconstruction methods for point-light and environment illu529 mination is shown side-by-side in Figures 19 and 20, respec530 tively. All differences are objectively compared using CIE $\Delta \mathrm{E}$, ${ }_{531}$ PSNR[dB], SSIM [30], and VDP2 [18] metrics. From the re532 sults it is apparent that for samples with lower height variations, ${ }_{533}$ there is a close match to the original data. The apparent devia534 tions from the original data for materials having higher surface ${ }_{535}$ height variations are caused mainly by the incorrect geometry 536 preservation of structural elements.

\section{7.2. Limitations}

${ }_{538}$ Although there are not any restrictions imposed on view and 539 illumination dependent datasets, the results have shown that ${ }_{540}$ the BTF reconstruction is incorrect for those materials which

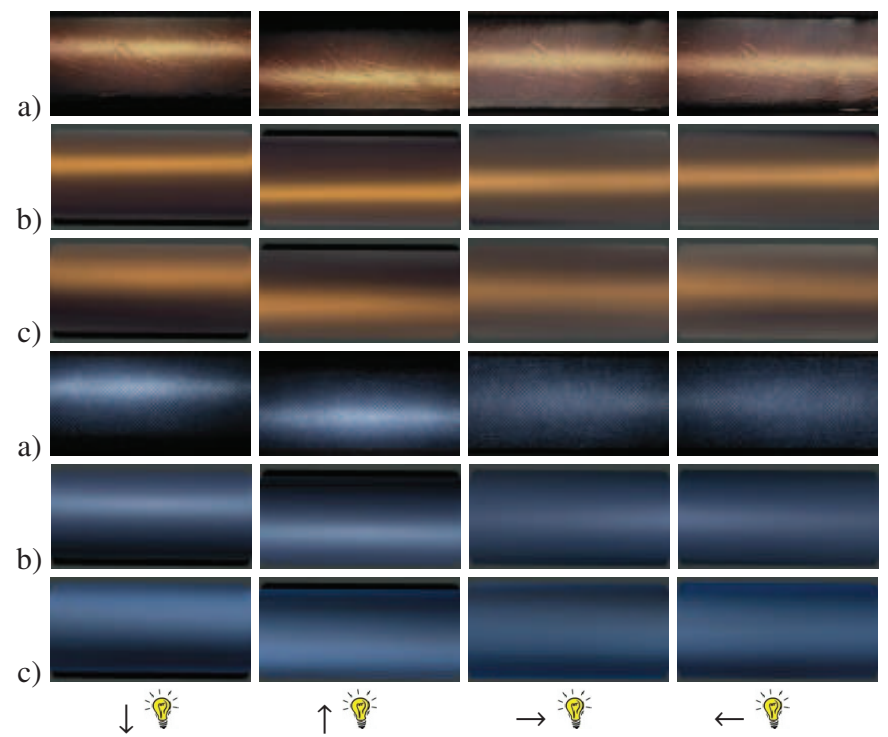

Figure 18: Photographs of the fabric01 and fabric02 samples on a cylinder illuminated from top, bottom, left, and right (a) compared with renderings using reference ABRDF (b), and sparsely measured and reconstructed ABRDF (c).

${ }_{541}$ have a wide range of surface height variation, e.g., corduroy 542 and Lego samples shown in Fig. 21. This is caused partly by 543 very sparse sampling of the azimuthal space, as well as by in544 terpolation of the data at missing elevations. While the former 545 produces geometrical deformation of the structure's features, 546 the latter causes their blur as well as improper highlights ex547 trapolation for low elevation angles. Even though the recon548 struction from sparse samples for such materials is not accurate ${ }_{549}$ in terms of correct shading of structure elements, the method 550 correctly captures the look-and-feel of the material's spatially551 varying appearance for nearly-flat samples, e.g., for fabric dark, 552 fabric light, and leather light samples. However, in compar553 ison with the SVBRDF measurement and representation ap${ }_{554}$ proaches, the proposed method is not limited to restrictions im555 posed by BRDF itself. Therefore, it may be found useful for 556 quick, low-cost, and fairly accurate acquisition and BTF recon557 struction of many materials having a limited height variation, 558 e.g., fabric and leather.

559 The time of BTF data reconstruction depends only on its 560 spatial resolution, since individual pixels are regarded as inde${ }_{561}$ pendent ABRDFs. Due to huge sizes of datasets, only repetitive ${ }_{562}$ BTF tiles were used. While reconstruction of a single pixel took $563 \approx 1$ second, the non-optimized reconstruction of a BTF tile of 564 size $128^{2}$ took 4.5 hours using the 3 cores of the Intel Xeon $5652.7 \mathrm{GHz}$. Therefore, using optimized multi-core CPU's imple566 mentation processing times of less than one hour can be easily 567 achieved.

568 Note that the proposed sparse acquisition and reconstruc569 tion method is complementary to BTF compression methods. 570 In always processing an entire BTF dataset, any of these meth571 ods can be applied to compress the reconstructed data. By its 572 sparse measurement, our method can achieve a compression ra573 tio 1:39; however, in terms of reconstruction quality and com574 pression ratio, it cannot compete with BTF compression ap575 proaches, as seen in the local PCA method [21] (using 5 clus- 


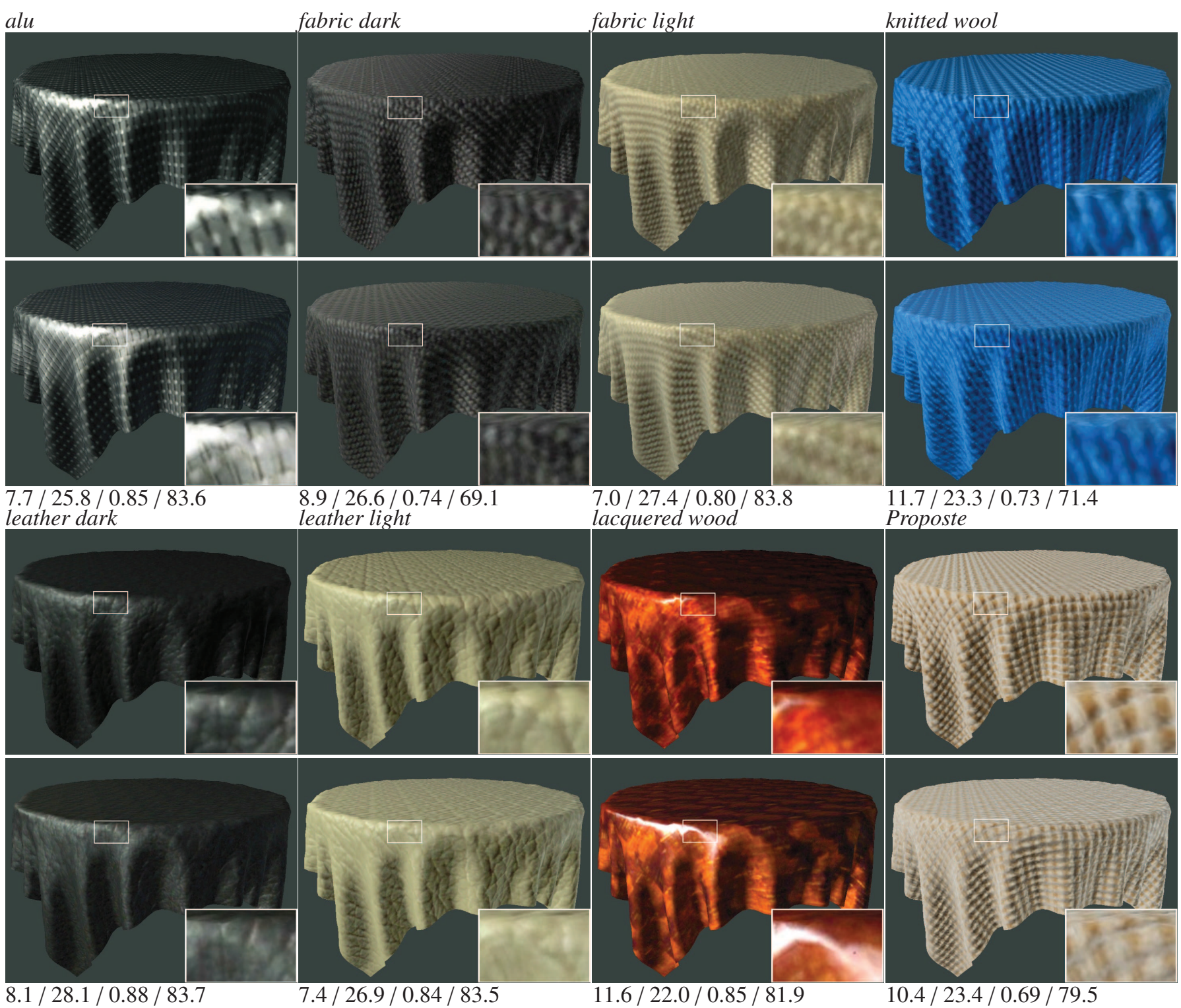

$8.1 / 28.1 / 0.88 / 83.7$

$7.4 / 26.9 / 0.84 / 83.5$

$11.6 / 22.0 / 0.85 / 81.9$

$10.4 / 23.4 / 0.69 / 79.5$

Figure 19: A comparison of BTF rendering from the full dataset of 6561 images (the first row), with its reconstruction from only 168 images (the second row) in single point-light illumination. Below are the CIE $\triangle \mathrm{E} / \mathrm{PSNR}[\mathrm{dB}]$ / SSIM / VDP2 difference values.

576 ters, 5 components) in Tab. 1 (compare with our reconstruction 577 errors in Fig. 19). The variable compression ratio of the local ${ }_{578}$ PCA method is due to the variable size of the BTF tile used.

\section{8. Conclusions}

580 A novel method of sparse measurement and reconstruction ${ }_{581}$ of view and illumination dependent datasets has been proposed. ${ }_{582}$ The proposed sparse sampling of illumination and viewing di583 rections allows for intuitive continuous measurement by a con584 sumer camera and LED light. The reconstruction from such 585 sparse data does not impose any restrictions on input data and 586 allows reliable approximation of anisotropic non-reciprocal view 587 and illumination dependent datasets. Additionally, this method 588 can provide arbitrarily dense data reconstruction of both incom589 ing and outgoing directions. The method's performance was 590 tested on isotropic BRDFs and anisotropic apparent BRDFs
Table 1: A reconstruction error and compression ratio of LPCA compression method.

\begin{tabular}{|c|c|c|c|c|c|c|}
\hline material & \multicolumn{4}{|c|}{$\Delta \mathrm{E} / \mathrm{PSNR}[\mathrm{dB}]$ / SSIM / VDP2 } & \multicolumn{2}{|c|}{ C.R. (tile) } \\
\hline alu & 3.5 & 34.6 & 0.99 & 93.8 & 18.2 & $(21 \times 26)$ \\
\hline corduroy & 2.7 & 37.5 & 0.97 & 93.6 & 55.0 & $(36 \times 46)$ \\
\hline fabric $d$. & 4.6 & 32.2 & 0.94 & 86.2 & 16.1 & $(21 \times 23)$ \\
\hline fabric $l$. & 8.6 & 26.3 & 0.95 & 89.4 & 10.4 & $(19 \times 23)$ \\
\hline leather $d$. & 4.3 & 33.3 & 0.97 & 91.0 & 263.0 & $(93 \times 86)$ \\
\hline leather $l$. & 10.8 & 24.3 & 0.95 & 87.7 & 193.0 & $(74 \times 79)$ \\
\hline l. wood & 11.3 & 23.5 & 0.92 & 81.0 & 627.8 & $(137 \times 142)$ \\
\hline k. wool & 4.3 & 32.1 & 0.96 & 93.0 & 20.8 & $(25 \times 25)$ \\
\hline
\end{tabular}




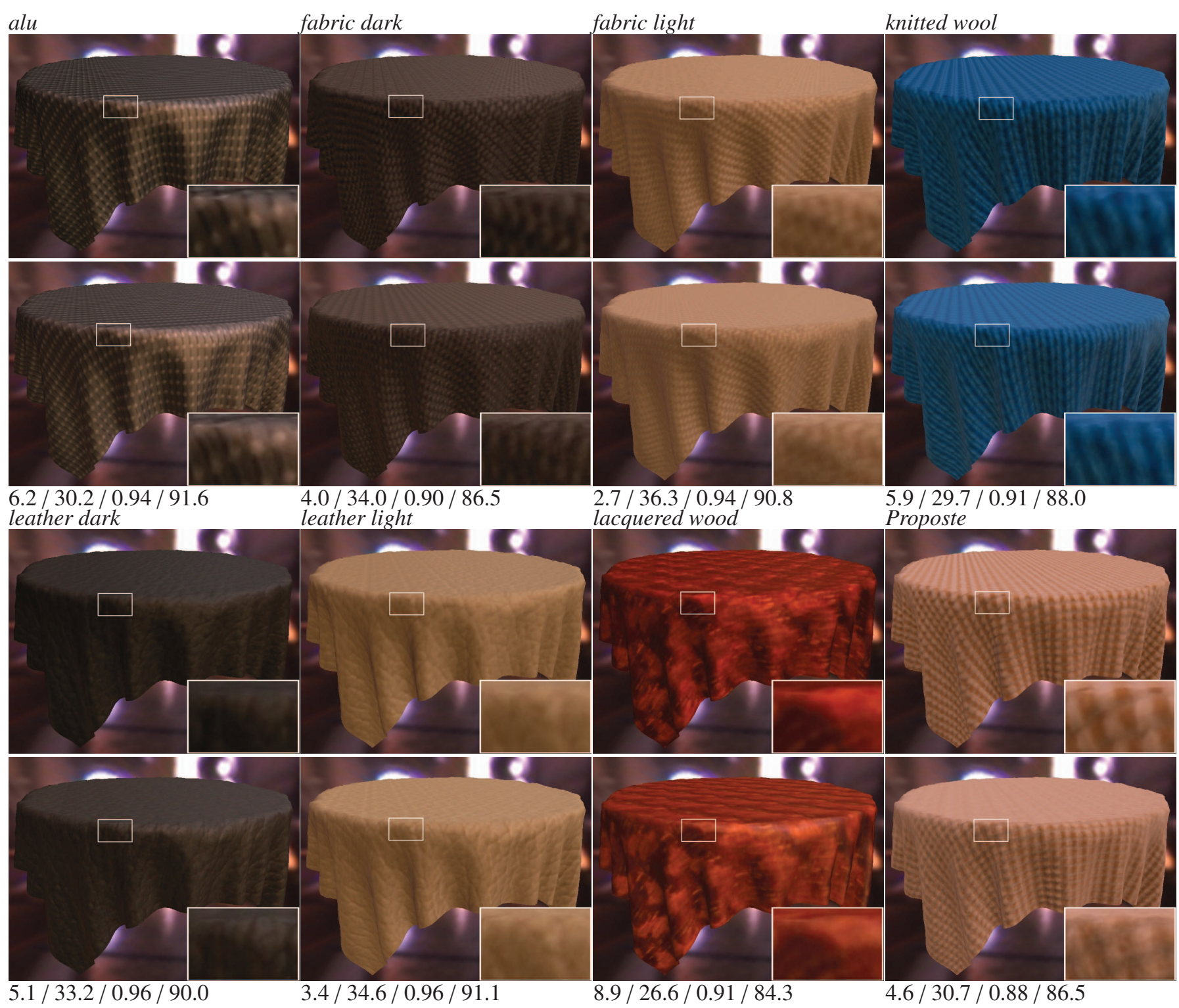

Figure 20: A comparison of BTF rendering from the full dataset of 6561 images (the first row), with its reconstruction from only 168 images (the second row) in grace environment illumination [5]. Below are the CIE $\Delta \mathrm{E}$ / PSNR[dB] / SSIM / VDP2 difference values.

591 with encouraging results. Our pilot ABRDF measurement ex592 periments have shown that retrieval of sparse samples and the 593 consequent reconstruction of the complete dataset take less than

594 half an hour. Experimental sparse reconstruction of BTF datasets 610 Acknowledgments

595 has shown that the method can be a reasonably accurate alter596 native to lengthy measurement, especially for samples having a 597 smaller height variation. The ease of data acquisition and visual 598 quality of the reconstruction using this method makes it supe599 rior to alternative approaches such as bump/displacement map600 ping or parametric BRDF modeling. Because of the simplicity 601 of data acquisition and reconstruction, this approximate method 602 can be utilized in less accuracy-demanding applications. Since 603 digital reproduction of a material's appearance look-and-feel 604 can be created inexpensively, it could be particularly useful in 605 the fields of computer gaming, film and digital presentations of 6 606 e-commerce.

607 In summation, we believe this research will contribute to 608 future development of simple, inexpensive, and portable acqui- 623
609 sition setups of illumination and view dependent data.

This work has been supported by the Czech Science Foun612 dation grant GAP 103/11/0335 and EC Marie Curie European 613 Reintegration Grant No 239294.

\section{${ }_{14}$ References}

[1] N. G. Alldrin, T. Zickler, and D. Kriegman. Photometric stereo with non-parametric and spatially-varying reflectance. In IEEE Conference on Computer Vision and Pattern Recognition (CVPR), pages 1-8, 2008.

[2] Nicolas Bonneel, Michiel van de Panne, Sylvain Paris, and Wolfgang Heidrich. Displacement interpolation using lagrangian mass transport. ACM Transactions on Graphics, 30(6), December 2011.

[3] K.J. Dana, B. van Ginneken, S.K. Nayar, and J.J. Koenderink. Reflectance and texture of real-world surfaces. ACM Transactions on Graphics, 18(1):1-34, 1999. 


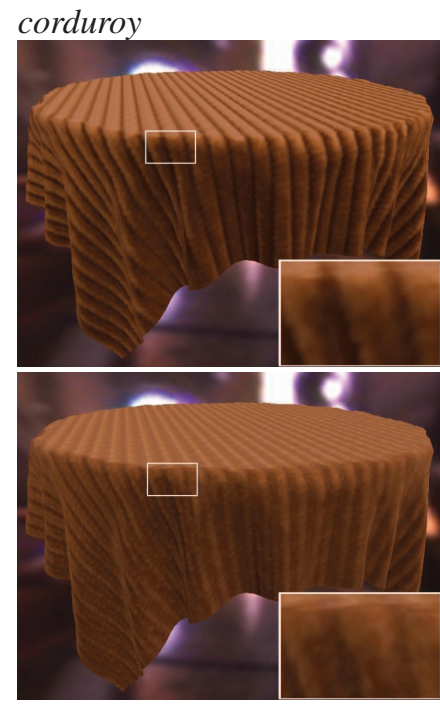

$7.6 / 28.1 / 0.82 / 74.5$
Lego

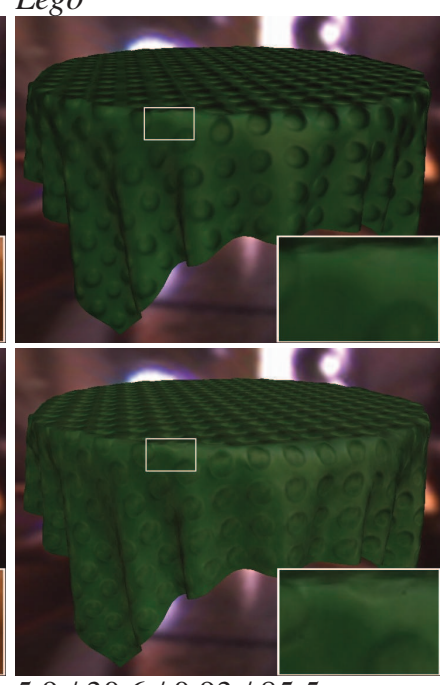

$5.9 / 30.6 / 0.93 / 85.5$

Figure 21: A failure case of the proposed BTF reconstruction for rough materials: corduroy and Lego. Below are the CIE $\triangle \mathrm{E} / \mathrm{PSNR}[\mathrm{dB}]$ / SSIM / VDP2 difference values.

[4] K.J Dana and J. Wang. Device for convenient measurement of spatially varying bidirectional reflectance. Journal of Optical Society of America, 21(1):1-12, 2004.

[5] Paul Debevec. Rendering synthetic objects into real scenes: bridging traditional and image-based graphics with global illumination and high dynamic range photography. In Proceedings of the 25th annual conference on Computer graphics and interactive techniques, SIGGRAPH '98, pages 189-198, 1998.

[6] Yue Dong, Jiaping Wang, Xin Tong, John Snyder, Yanxiang Lan, Moshe Ben-Ezra, and Baining Guo. Manifold bootstrapping for SVBRDF capture. ACM Transactions on Graphics, 29(4):98:1-98:10, 2010.

[7] J. Filip. Restoring illumination and view dependent data from sparse samples. In Proceedings of the 21th International Conference on Pattern Recognition, ICPR 2012, pages 1391-1394, November 2012.

[8] A. Ghosh, T. Chen, P. Peers, C.A. Wilson, and P. Debevec. Circularly polarized spherical illumination reflectometry. ACM Transactions on Graphics, 29(6):162:1-162:12, 2010.

[9] D. B. Goldman, B. Curless, A. Hertzmann, and S. M. Seitz. Shape and spatially-varying BRDFs from photometric stereo. In Proceedings of ICCV, ICCV '05, pages 341-348, 2005.

44 [10] J.Y. Han and K. Perlin. Measuring bidirectional texture reflectance with a kaleidoscope. ACM SIGGRAPH 2003, 22(3):741-748, July 2003.

646 [11] V. Havran, J. Filip, and K. Myszkowski. Bidirectional texture function compression based on multi-level vector quantization. Computer Graphics Forum, 29(1):175-190, jan 2010.

49 [12] M. Holroyd, J. Lawrence, and T. Zickler. A coaxial optical scanner for synchronous acquisition of 3D geometry and surface reflectance. ACM Transactions on Graphics, 29(4):99:1-99:12, 2010.

2 [13] M. B. Hullin, J. Hanika, B. Ajdin, H.-P. Seidel, J. Kautz, and H. P. A. Lensch. Acquisition and analysis of bispectral bidirectional reflectance and reradiation distribution functions. ACM Transactions on Graphics, 29(4):1-7, 2010.

[14] M. Kurt, L. Szirmay-Kalos, and J. Křivánek. An anisotropic BRDF model for fitting and monte carlo rendering. SIGGRAPH Comput. Graph., 44:3:1-3:15, February 2010.

59 [15] E. P. Lafortune, S. Ch. Foo, K. E. Torrance, and D. P. Greenberg. Nonlinear approximation of reflectance functions. Computer Graphics Forum, 31:117-126, 1997.

62 [16] J. Lawrence, A. Ben-Artzi, C. DeCoro, W. Matusik, H. Pfister, R. Ramamoorthi, and S. Rusinkiewicz. Inverse shade trees for non-parametric material representation and editing. ACM Transactions on Graphics, 25(3):735-745, 2006.

66 [17] H. P.A. Lensch, J. Lang, A. M. Sá, and H.-P. Seidel. Planned sampling of spatially varying BRDFs. Computer Graphics Forum, 22(3):473-482, 2003.

[18] Rafat Mantiuk, Kil Joong Kim, Allan G. Rempel, and Wolfgang Heidrich. Hdr-vdp-2: a calibrated visual metric for visibility and quality predictions in all luminance conditions. ACM Transactions on Graphics, 30(4):40:140:14, 2011.

[19] W. Matusik, H. Pfister, M. Brand, and L. McMillan. A data-driven reflectance model. ACM Transactions on Graphics, 22(3):759-769, 2003.

5 [20] G. Müller, G.H. Bendels, and R. Klein. Rapid synchronous acquisition of geometry and BTF for cultural heritage artefacts. In The 6th International Symposium on Virtual Reality, Archaeology and Cultural Heritage (VAST), pages 13-20, 2005.

[21] G. Müller, J. Meseth, and R. Klein. Compression and real-time rendering of measured BTFs using local PCA. In Vision, Modeling and Visualisation, pages 271-280, 2003.

2 [22] A. Ngan and F. Durand. Statistical acquisition of texture appearance. 17th Eurographics Symposium on Rendering, pages 31-40, August 2006.

4 [23] A. Ngan, F. Durand, and W. Matusik. Experimental analysis of BRDF models. Eurographics Symposium on Rendering 2005, 2:117-126, 2005.

6 [24] A. Ngan, F. Durand, and W. Matusik. Image-driven navigation of analytical BRDF models. Proceedings of the Eurographics Symposium on Rendering, pages 399-407, 2006.

[25] F.E. Nicodemus, J.C. Richmond, J.J. Hsia, I.W. Ginsburg, and T. Limperis. Geometrical considerations and nomenclature for reflectance. NBS Monograph 160, National Bureau of Standards, U.S. Department of Commerce, pages 1-52, 1977.

[26] W. H. Press, S. A. Teukolsky, William T. Vetterling, and Brian P. Flannery. Numerical Recipes in C: The Art of Scientific Computing. Cambridge University Press, 1992.

[27] Peiran Ren, Jiaping Wang, John Snyder, Xin Tong, and Baining Guo. Pocket reflectometry. ACM Transactions on Graphics, 30(4):45:1-45:10, 2011.

[28] S.M. Rusinkiewicz. A new change of variables for efficient BRDF representation. In Rendering techniques' 98, pages 11-22, 1998.

[29] M. Sattler, R. Sarlette, and R. Klein. Efficient and realistic visualization of cloth. In Eurographics Symposium on Rendering 2003, pages 167-178, 2003.

[30] Z. Wang, A.C. Bovik, H.R. Sheikh, and E.P. Simoncelli. Image quality assessment: From error visibility to structural similarity. IEEE Transactions on Image Processing, 13(4):600-612, 2004.

[31] Z. Zhengyou. Flexible camera calibration by viewing a plane from unknown orientations. In In proceedings of ICCV, pages 666-673, 1999.

99 [32] T. Zickler, R. Ramamoorthi, S. Enrique, and P. N. Belhumeur. Reflectance sharing: Predicting appearance from a sparse set of images of a known shape. IEEE PAMI, 28(8):1287-1302, 2006. 